\title{
Design and Optimization of Piezoelectric Dual-Mode Micro-Mirror
}

\author{
Jichao Zhong, Xingguo Xiong, Zheng Yao, Junling Hu*, Prabir Patra* \\ Department of Electrical and Computer Engineering, \\ *Department of Mechanical Engineering \\ University of Bridgeport, Bridgeport, CT 06604, USA
}

\begin{abstract}
MEMS micro-mirrors have been widely used in optical communication, projection display, and microscanner, etc. Various MEMS micro-mirror devices have been reported. In this paper, the design of a bulkmicromachined MEMS piezoelectric dual-mode micromirror is proposed. The working principle of the dualmode micro-mirror device is analyzed. Based on the analysis, a set of optimized design parameters of the micro-mirror is suggested. Theoretical analysis shows that the proposed micro-mirror can deflect for a maximum displacement of $2.5 \mu \mathrm{m}$ in its piston mode, and tilt for a maximum angle of $\mathbf{4 . 8 8}^{\circ}$ in its torsional mode. The fabrication flow of the MEMS micro-mirror is also suggested. The proposed micro-mirror has the advantages of large displacement and large tilt angle due to piezoelectric activation. The PZT actuated micromirror (PAM) also leads to improved linearity in tilting angle and piston displacement. Further, fast response of the PZT unimorph on the applied actuation voltage ensures wider operation bandwidth of the PAM in contrast to thermal activated micro-mirrors.
\end{abstract}

Keywords: MEMS (Microelectromechanical System), dualmode micro-mirror, piezoelectric technology (PZT), piston micro-mirror, torsional micro-mirror.

\section{Introduction}

Microelectromechanical Systems (MEMS) deal with devices or systems in the size range of $1 \mu \mathrm{m} \sim 1 \mathrm{~mm}$, which integrates both mechanical and electrical components into a single chip. MEMS merges with nanotechnology and creates the field of Nanoelectromechanical Systems (NEMS). Optical MEMS is a unique application where MEMS are used to direct, guide, filter, and, in some instances, amplify light. Well known applications of optical MEMS include optical switching and digital light projection. In optical switching applications, micro-mirrors are used to steer light from an incoming fiber optic input to a fiber optic output. In digital light projection applications, micro-mirrors, such as digital micro-mirror device (DMD) by Texas Instruments Inc., incident light is reflected by an array of torsional micro-mirrors so that each mirror pixel can control a corresponding spot on the screen to be bright or dark. In this way, vivid pictures can be projected onto the screen.
According to the working modes, MEMS micro-mirrors can be divided into two categories: piston mirrors and torsional mirrors. Piston micro-mirrors move perpendicular to the device plan, while torsional micro-mirrors rotate along a certain axis. Various piston and torsional micro-mirrors have been reported [1]-[4]. The working principles and design considerations of MEMS micromirrors have also been analyzed in details [5]-[6]. Furthermore, dual-mode micro-mirrors which can work in both piston and torsional modes have been reported [7]-[8]. Dual-mode micro-mirrors offers more flexibility in light modulation. Users can reconfigure them into either piston or torsional modes according to their individual needs.dd

MEMS micro-mirrors can be activated using various mechanisms, such as electrostatic driving, electromagnetic driving, thermal driving, piezoelectric driving, as well as shape-memory alloy driving, etc. Among them, electrostatic driving is popular due to their easy in implementation. But the maximum displacement is limited by snap-down effect in perpendicular driving. Electromagnetic driving has larger activation force and displacement, but the fabrication is more complex. Thermal driving is relatively slow in response, and the energy efficiency is not high due to thermal dissipation. Piezoelectric driving utilizes piezoelectric effect to activate MEMS micro-mirrors. It can achieve large displacement or rotation angle with high energy efficiency. Various piezoelectric micro-mirrors have been reported [9]-[11].

In this paper, a bulk-micromachined piezoelectric-driven, dual-mode MEMS micro-mirror device is proposed. The dual-mode micro-mirror can be configured to work in either piston or torsional mode according to the need. The working principle of the dual-mode micro-mirror device is analyzed. Based on the analysis, a set of optimized design parameters of the micro-mirror is suggested. Theoretical analysis shows that the proposed micro-mirror can deflect for a maximum displacement of $2.5 \mu \mathrm{m}$ in its piston mode, and tilt for a maximum angle of $4.88^{\circ}$ in its torsional mode. The fabrication flow of the MEMS micro-mirror is also suggested. The proposed micro-mirror can achieve large displacement and large tilt angle due to piezoelectric activation. The PZT actuated micro-mirror (PAM) also has improved linearity in tilting angle and piston displacement.

\section{Piezoelectric Activation}


Piezoelectric materials are used for both sensing and actuation purposes. It was observed that certain materials generate an electric charge (or voltage) when they are under mechanical stress. This is known as the direct effect of piezoelectricity. Alternately, the same materials would be able to produce a mechanical deformation (or force) when an electric field is applied to them. This is called the inverse effect of piezoelectricity.

Piezoelectric materials are crystals. The microscopic origin of piezoelectricity is the displacement of ionic charges within a crystal, leading to the polarization and electric field. A stress (tensile or compressive) applied to a piezoelectric crystal will alter the spacing between centers of positive and negative charge sites in each domain cell; this leads to a net polarization manifested as open circuit voltages measurable at the crystal surface. Compressive and tensile stresses will generate electric fields and hence voltages of opposite polarity.

Inversely, an external electric field will exert a force between the centers of positive and negative charges, leading to an elastic strain and changes of dimensions depending on the field polarity. Not all naturally occurring or synthesized crystals exhibit piezoelectricity. Crystals can be classified into 32 groups according to crystal symmetry. Centrosymmetric crystal structures are crystals that are symmetric along all axes through the center of the crystal. These crystals occupy 11 out of 32 possible groups and are non-piezoelectric materials because the positive and negative charge sites will not be spatially separated under stress. Out of 21 non-centrosymmetric groups, 20 are piezoelectric crystals. Piezoelectric effects are strongly orientation dependent. The notation conventions for crystal orientations in the context of piezoelectric polarization are discussed first. A piezoelectric material needs to be poled in a particular direction to provide a strong piezoelectric effect, although some materials exhibit natural or spontaneous polarization. The direction of positive polarization is customarily chosen to coincide with the $Z$-axis of a rectangular system of crystallographic axes $\mathrm{X}, \mathrm{Y}$, and $\mathrm{Z}$. Alternatively, the normal stress components along axes $X$, $\mathrm{Y}$, and $\mathrm{Z}$ are denoted by subscripts 1,2 and 3, respectively, as shown in Figure 1. As such, the poling axis always coincides with axis-3. Shear stress and strain components about these axes are denoted by sub-scripts 4,5 and 6 respectively.

In a piezoelectric crystal, the constitutive equation that relates electrical polarization (D) and applied mechanical stress $(\mathrm{T})$ is [12]:

$$
D=d T+\varepsilon E
$$

where $\mathrm{d}$ is the piezoelectric coefficient matrix, $\varepsilon$ is the electrical permittivity matrix, and $\mathrm{E}$ is the electrical field. Here, an electric field is applied in conjunction with the mechanical stress to provide more generality. The electrical polarization is contributed by two parts - one stemming from electrical biasing and one from mechanical loading.
If no electric field is present (i.e., $\mathrm{E}=0$ ), then the second term on the right-hand side of the above equation can be eliminated.

The general constitutive equation can be written in the full matrix form:

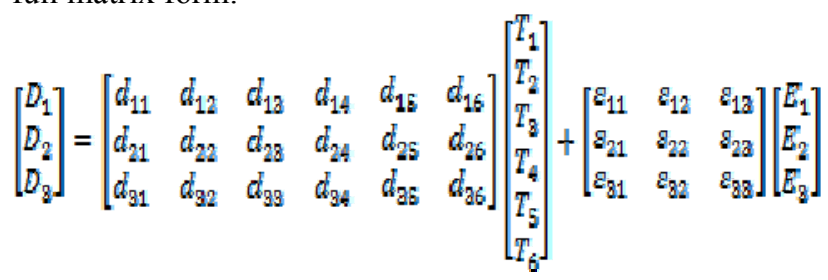

the terms $T_{1}$ through $T_{3}$ are normal stresses along axes 1,2 and 3 , whereas $T_{4}$ through $T_{6}$ are shear stresses. The units of electrical displacement $\left(\mathrm{D}_{\mathrm{i}}\right)$, stress $\left(\mathrm{T}_{\mathrm{i}}\right)$, permittivity $\left(\varepsilon_{\mathrm{i}}\right)$, and electrical field $\left(E_{j}\right)$ are $C / \mathrm{m}^{2}, \mathrm{~N} / \mathrm{m}^{2}, \mathrm{~F} / \mathrm{m}^{2}$, and $\mathrm{V} / \mathrm{m}$, respectively. The unit of the piezoelectric constant $d_{i j}$ is the unit of electric displacement divided by the unit of the stress, namely:

$$
\left[d_{i j}\right]=\frac{[D]}{[T]}=\frac{[\varepsilon][E]}{[T]}=\frac{\frac{F}{m} \frac{V}{m}}{\frac{N}{m^{2}}}=\frac{\text { Columb }}{N}
$$

3

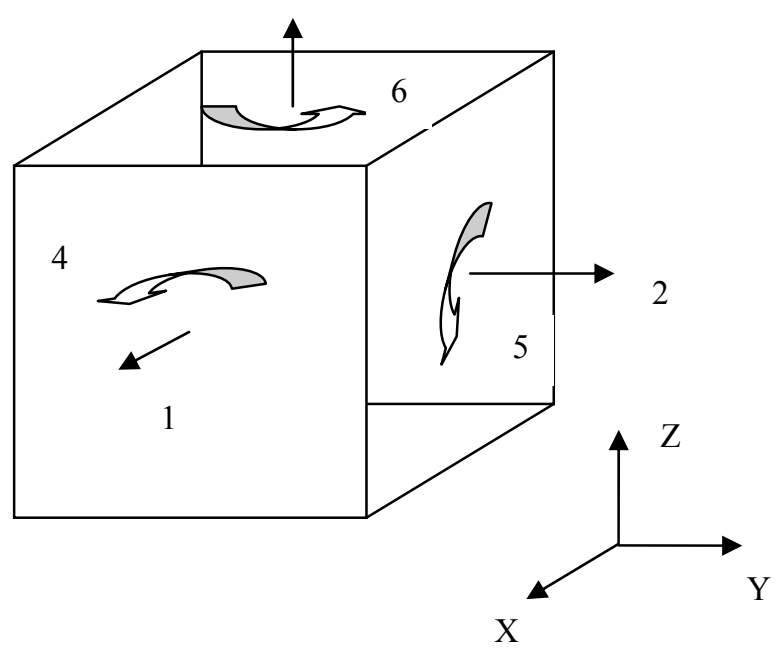

Figure 1: Schematic illustration of a piezoelectric crystal in a Cartesian coordinate system [12]

The inverse effect of piezoelectricity can be similarly described by a matrix-form constitutive equation. In this case, the total strain is related to both the applied electric field and any mechanical stress [12], according to

$$
s=S T+d E
$$

Where $\mathrm{s}$ is the strain vector and $\mathrm{S}$ is the compliance matrix. 
This equation can be expanded to a full matrix form [12]:

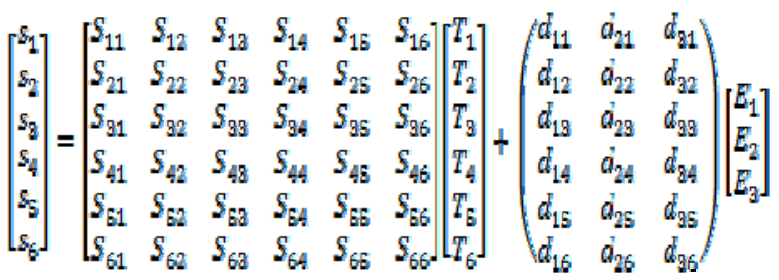

if there is no mechanical stress present $\left(\mathrm{T}_{\mathrm{i}, \mathrm{i}=1,6}=0\right)$, the strain is related to the electric field by [12]

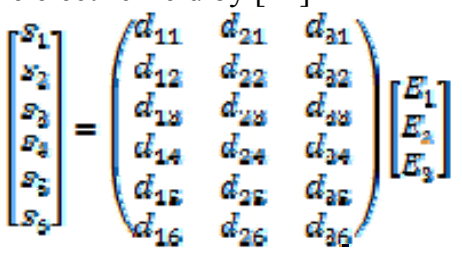

Note that, for any given piezoelectric material, the $d_{i j}$ components connecting the strain and the applied field in the inverse effect are identical to the $d_{i j}$ connecting the polarization and the stress in the direct effect.

The electromechanical coupling coefficient $\mathrm{k}$ is a measure of how much energy is transferred from electrical to mechanical energy, or vice versa, during the actuation process [12]:

$$
k^{2}=\frac{\text { energy_converted }}{\text { input_energy }}
$$

this relation holds true for both mechanical-to-electrical and electrical-to-mechanical energy conversion. The magnitude of $\mathrm{k}$ is a function of not only the material, but also the geometries of the sample and its oscillation mode.

\section{Design and Optimization of the Micro-mirror}

The schematic diagram of the proposed dual-mode piezoelectric micro-mirror is shown in Figure 1. ANSYS FEM simulation is used to verify the device function, as shown in Figure 2. As shown in Figure 1 and 2, a MEMS micro-mirror is connected to four torsional beams which extrudes from two torsional axes. On two of the four torsional beams, PZT piezoelectric materials are deposited to activate the torsional beams. When electrical driving voltages are applied to the PZT actuators on torsional beams, the PZT will expand or shrink, which generates corresponding torque to activate the micromirror to rotate/tilt along the torsional axes. In this way, the micromirror works in torsional mode. The two torsional axes are again connected to the outer frame, which are anchored to the substrate through four flexible piston beams. PZT actuators are also deposited on top of the four piston beams. When the electrical driving voltages are simultaneously applied to the four PZT actuators on the four piston beams, the PZT actuators will expand or shrink simultaneously, which in turn activates the frame (and the mirror) to move perpendicular to the device plane. Hence, the micro-mirror works in piston mode. By applying electrical driving voltages to the PZT actuators on either the piston or torsional beams, the micro-mirror can work in either piston or torsional mode. Hence this is a piezoelectric activated dual-mode micro-mirror design.

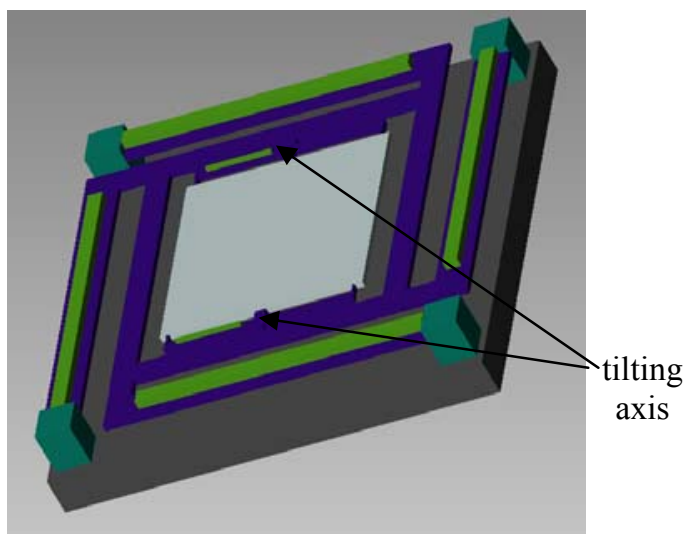

Figure 2. Schematic diagram of the dual-mode piezoelectric micro-mirror

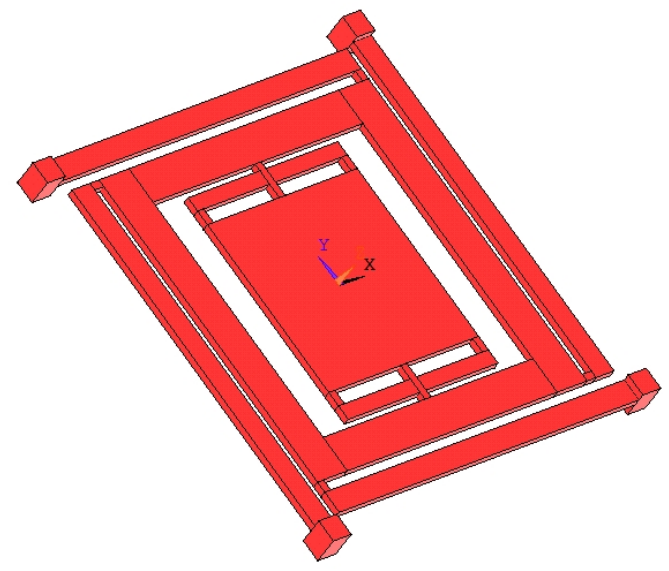

Fig 3: ANSYS model of the dual-mode micro-mirror

In this mirror design, PZT actuated micro-mirror (PAM) can provide improved linearity tilting angle and piston displacement. In addition, its displacement in piston mode is not limited by pull-in phenomenon as in perpendicular electrostatic actuation. Fast response of the PZT unimorph membrane on the applied actuation voltage ensures wider operation bandwidth of the PAM in contrast to the thermal activation. In this work, PZT-4 is chosen as piezoelectric material and polysilicon is used as substrate material for PZT actuators.

In this piezoelectric actuated micro-mirror, cantilever piezoelectric actuator model is used to analyze the device 
behavior. Piezoelectric actuators are often used in conjunction with cantilevers or membranes for sensing and actuation purpose. General models for such piezoelectric actuators are rather complex. Accurate analysis often involves finite element modeling. For limited cases, such as a cantilever actuator with two layers, analytical solution has been successfully achieved. Based on the theoretical analysis, a set of optimized design parameters of the micromirror is achieved, as shown in Table 1.

Table 1. Optimized design parameters of the dual-mode micro-mirror

\begin{tabular}{|l|l|l|}
\hline \multirow{4}{*}{ Outer actuator beam } & Length & $100 \mu \mathrm{m}$ \\
\cline { 2 - 3 } & Width & $6 \mu \mathrm{m}$ \\
\cline { 2 - 3 } & Thickness & $2 \mu \mathrm{m}$ \\
\hline \multirow{4}{*}{ Main frame (square) } & Length of side & $90 \mu \mathrm{m}$ \\
\cline { 2 - 3 } & Width of side & $10 \mu \mathrm{m}$ \\
\cline { 2 - 3 } & Thickness & $4 \mu \mathrm{m}$ \\
\hline Torsional axis & Length & $10 / 20 \mu \mathrm{m}$ \\
\cline { 2 - 3 } & Width & $2 \mu \mathrm{m}$ \\
\cline { 2 - 3 } & Thickness & $2 \mu \mathrm{m}$ \\
\hline \multirow{3}{*}{$\begin{array}{l}\text { Inner actuator beam } \\
\text { Length }\end{array}$} & $24 \mu \mathrm{m}$ \\
\cline { 2 - 3 } & Width & $4 \mu \mathrm{m}$ \\
\cline { 2 - 3 } $\begin{array}{l}\text { Gap between the outer } \\
\text { actuator beam and the } \\
\text { main frame }\end{array}$ & $2.5 \mu \mathrm{m}$ & $2 \mu \mathrm{m}$ \\
\hline $\begin{array}{l}\text { Gap between the inner } \\
\text { actuator beam and the } \\
\text { mirror }\end{array}$ & $2.5 \mu \mathrm{m}$ \\
\hline $\begin{array}{l}\text { Gap between the mirror } \\
\text { and the substrate }\end{array}$ & $3 \mu \mathrm{m}$ \\
\hline
\end{tabular}

The deflection of a two-layer piezoelectric structure can be derived based on above model. Consider a cantilever with two layers, one elastic and one piezoelectric, joined along one side. Assume the two layers have the same length. The beam bends into an arc when the piezoelectric layer is subjected to a longitudinal strain, $\mathrm{s}_{\text {long. The radius of }}$ curvature can be found by [12]

$$
\frac{1}{r}=\frac{2\left|s_{\text {long }}\right|\left(t_{p}+t_{e}\right)\left(A_{p} E_{p} A_{e} E_{e}\right)}{4\left(E_{p} I_{p}+E_{e} I_{e}\right)\left(A_{p} E_{p}+A_{e} E_{e}\right)+\left(t_{e}+t_{p}\right)^{2} A_{e} E_{e} A_{p} E_{p}}
$$

In which $A_{p}$ and $A_{e}$ are the cross-sectional area of the piezoelectric and the elastic layer; $E_{p}$ and $E_{e}$ are Young's modulus of the piezoelectric layer and Young's modulus of the elastic layer; and $t_{p}$ and $t_{e}$ are the thickness of the piezoelectric layer and the elastic layer.

The amount of force achievable at the free end of a piezoelectric bimorph actuator equals the force required to restore the tip of the actuator to its initial un-deformed state. Since the displacement is linearly related to force according to
$\delta(L)=F / k$

the force can be expressed as

$$
F=\delta(x=L) \cdot k
$$

In this work, PZT-4 is chosen as the piezoelectric material. For PZT-4,

$$
\begin{aligned}
& s=\left[\begin{array}{cccccc}
12.5 & -4.05 & -5.31 & 0 & 0 & 0 \\
-4.05 & 12.3 & -5.31 & 0 & 0 & 0 \\
-5.31 & -5.31 & 15.5 & 0 & 0 & 0 \\
0 & 0 & 0 & 89 & 0 & 0 \\
0 & 0 & 0 & 0 & 39 & 0 \\
0 & 0 & 0 & 0 & 0 & 3.9
\end{array}\right] \times 10^{-12} \mathrm{~m}^{2} / N \\
& d-\left[\begin{array}{cccccc}
0 & 0 & 0 & 0 & 496 & 0 \\
0 & 0 & 0 & 4 \% 6 & 0 & 0 \\
-125 & -122 & 28 \% & 0 & 0 & 0
\end{array}\right] \\
& s_{p}=\left[\begin{array}{ccc}
1475 & 0 & 0 \\
0 & 14.75 & 0 \\
0 & 0 & 1300
\end{array}\right]
\end{aligned}
$$

According to the structure we used for the actuator, we should choose $d_{31}$ as the piezoelectric parameter. So the displacement can be calculated as below:

$$
\begin{gathered}
\delta(x)=\frac{x^{2} d_{31}\left(t_{p}+t_{e}\right)\left(A_{p} E_{p} A_{e} E_{e}\right)}{4\left(E_{p} I_{p}+E_{e} I_{e}\right)\left(A_{p} E_{p}+A_{e} E_{e}\right)+\left(t_{e}+t_{p}\right)^{2} A_{e} E_{e} A_{p} E_{p}} \\
=1.05 \times 10^{-6} \times V(\mu \mathrm{m})
\end{gathered}
$$

Under small-deflection approximation, the dual-mode micro-mirror can be treated as simplified spring-mass model. In the piston mode, the four piston beams can be modeled as double-clamped beams. The spring constant of one double-clamped beam can be calculated as

$$
K_{b}=\frac{12 E I_{b}}{L_{b}^{3}}
$$

Hence in our design, the displacement at the end of the piston beam can be calculated as

$$
\delta(x)=\frac{1.05 \times 10^{-6} \times V}{4}=0.25 \mathrm{~V}(\mu \mathrm{m})
$$

The designed driving voltage of this micro-mirror is $10 \mathrm{~V}$, so the maximum displacement of the micro-mirror in piston mode is $2.5 \mu \mathrm{m}$.

When the driving electrical voltage is applied to the PZT actuators in the inner torsional beams, the PZT actuators will generate corresponding torque on the torsional beams, hence the micro-mirror will tilt along the torsional axes. As a result, the dual-mode micro-mirror works in torsional mode. Assume the voltage is $50 \mathrm{~V}$, and the length of the torsional axis is $2 \mu m(h) \times 2 \mu m(b) \times 4 \mu m(l)$, based on the above design parameters, the displacement of the actuator is calculated to be

$$
\delta(x)=0.975(\mu m)
$$

The tilt angle $\theta$ is calculated to be

$$
\theta=2.32^{\circ}
$$

we can optimize the mirror design on inner actuator, the actuator will counteract the torque of the torsional axis, and the tilt angle of the torsional axis must follow this equation: 


$$
\varphi=\frac{T l}{G B h b^{3}}
$$

where $T$ is torque; 1 is the length of the torsional axis, $G$ is shear modulus (for polysilicon, $\mathrm{G}=69 \mathrm{GPa}$ ), $\beta$ is a constant, $h$ is the height of torsional beam, and $b$ is the width of the torsional beam. Now, the size of the torsional axis can be redesigned as $2 \mu \mathrm{m}(\mathrm{h}) \times 2 \mu \mathrm{m}(\mathrm{b}) \times 4 \mu \mathrm{m}(\mathrm{l})$. For $\beta=0.141$, under the same torque, the displacement of the micro-mirror is found to be in linear relationship with the torsional driving voltage:

$$
\delta(x)=0.041 V(\mu m)
$$

For a torsional driving voltage of $50 \mathrm{~V}$, the corresponding displacement and the torsional angle of the micromirror are found to be

$$
\begin{aligned}
& \delta(x)=2.05(\mu \mathrm{m}) \\
& \theta=4.88^{\circ}
\end{aligned}
$$

\section{Fabrication Flow}

A fabrication process of the above dual-mode micromirror is illustrated in Figure 4. Starting from a bare silicon wafer, we coat the top side of the silicon wafer with $2 \mu \mathrm{m}$ thickness LPCVD low stress silicon nitride. In order to pattern the PZT layer, a thin layer of $150 \mathrm{~nm}$-thick platinum layer is pre-deposited at the bottom electrode layer. Then a $0.2 \mu \mathrm{m}$-chick PZT-4 layer is deposited using sol-gel method. On the top of the PZT-4 layer, we deposited a $150 \mathrm{~nm}$-thick ruthenium oxide layer. This forms the PZT piezoelectric actuator structures for the micro-mirror.

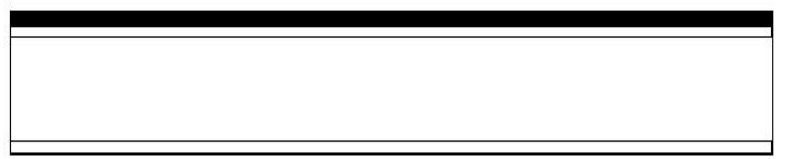

(a) Silicon wafer coated with $\mathrm{Si}_{3} \mathrm{~N}_{4}$ and PZT film

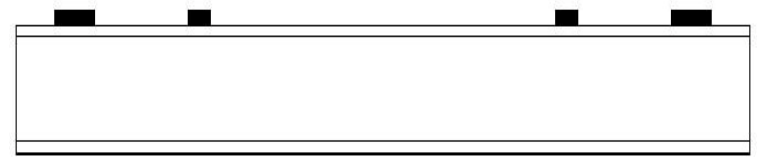

(b) Patterning of the PZT layer is delineated by self-aligned process using $\mathrm{Cl} / \mathrm{O}$-based plasma etching.

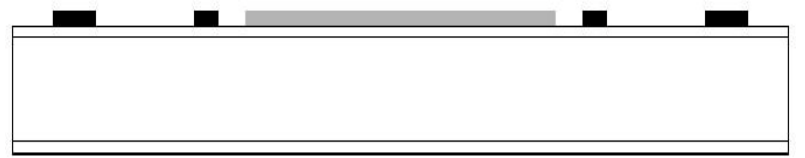

(c) The aluminum mirror surface is patterned by lift-off process.

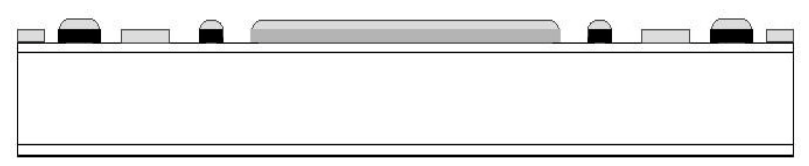

(d) Thin hard mask layer is deposited and patterned.

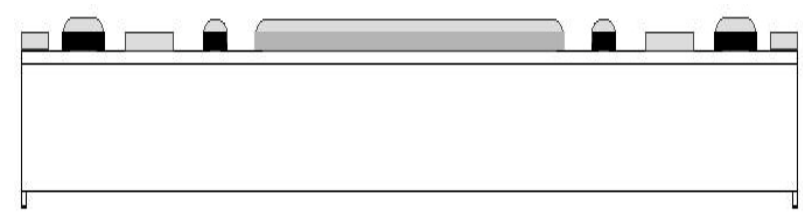

(e) The silicon nitride at the backside of the wafer is patterned by double side aligning and subsequent RIE to define the etch window for silicon bulk etching.

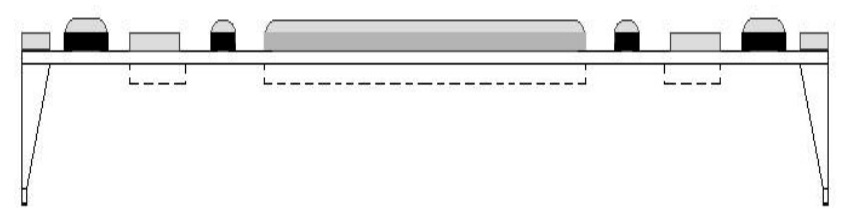

(f) The silicon under the suspended microstructures is removed by anisotropic etching in aqueous $\mathrm{KOH}$. The movable microstructure can be released to be free-standing.

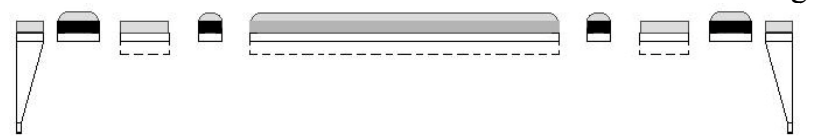

(g) Suspended structures including the mirror plate are released by RIE from the front side of the wafer using the patterned metal film at the step (d) as the masking material.

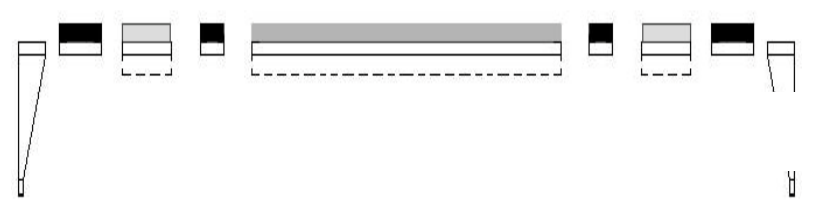

(h) Finally, released mirror are diced into individual chips after removing the hard mask metal

Figure 4. Fabrication flow of the piezoelectric dual-mode micromirror

\section{Conclusions and Future Work}

In this paper, a piezoelectric actuated dual-mode micromirror design is proposed. The working principle of the micro-mirror is analyzed. The micro-mirror is actuated by four PZT actuator beams in piston mode. The inner set of two PZT actuators is used to tilt the micro-mirror in torsional mode. Each set of actuators can work independently. Based on the analysis, a set of optimized design parameters of the micro-mirror is achieved. The two modes (piston and torsional) can be use for different situations as needed. Theoretical analysis shows that the proposed micro-mirror can deflect for a maximum displacement of $2.5 \mu \mathrm{m}$ in its piston mode, and tilt for a maximum angle of $4.88^{\circ}$ in its torsional mode. The fabrication flow of the MEMS micro-mirror is also suggested. The proposed micro-mirror can achieve large displacement and large tilt angle due to piezoelectric 
activation. The PZT actuated micro-mirror (PAM) also has improved linearity in tilting angle and piston displacement.

In the future work, we will further improve the structure design, so that the driving voltage can be reduced from current $10 \mathrm{~V}$ in piston mode to around $5 \mathrm{~V}$. In this way, it can be compatible with the power supply of on-chip CMOS circuitry. By reducing the required driving voltage, the power consumption of the micro-mirror can also be further reduced.

\section{References}

[1] G. Zhou, V.J. Logeeswaran, F.E.H. Tay, F.S. Chau, "Digital deflection micro-mirrors for adaptive optics applications", 12th International Conference on Solid-State Sensors, Actuators and Microsystems (Transducers'03), Vol. 1, June 8-12, 2003, pp. a591-a594.

[2] K. Jia, S. Pal, H. Xie, "An electrothermal tip-tilt-piston micromirror based on folded dual S-shaped bimorphs", Journal of Microelectromechanical Systems, Vol. 18, Issue 5, Oct. 2009, pp. 1004-1015.

[3] G. Vdovin, S. Middelhoek, L. Sarro, "Deformable mirror display with continuous reflecting surface micromachined in silicon", IEEE Proceedings of Micro Electro Mechanical Systems (MEMS'95), Jan. 29 - Feb. 2, 1995, pp. 61-65.

[4] K.B. Lee, L. Lin, "A vertically-supported two-axial torsional micro-mirror", Proceedings of 17th IEEE International Conference on Micro Electro Mechanical Systems (MEMS'04), 2004, pp. 41-44.

[5] O. Degani, Y. Nemirovsky, "Design considerations of rectangular electrostatic torsion actuation based on new analytical pull-in expression”, Journal of Microelectromechanical Systems, Vol. 11, pp. 20-26, 2002. [6] X. M. Zhang, F. S. Chau, C. Quan, Y. L. Lam, A. Q. Liu, "A study of the static characteristics of a torsional micro-mirror", Sensors and Actuators A: Physical, Vol. 90, 2001, pp. 73-81.

[7] U. Krishnamoorth, K. Li, K. Yu, D. Lee, J. P. Heritage, O. Solgaard, "Dual-mode micro-mirrors for optical phased array applications", Sensors and Actuators A: Physical, Vol. 97-98, 2002, pp. 21-26.

[8] X. Xiong, T. Dallas, S. Gangopadhyay, J. Berg, T. Henryk, "Design and Simulation of Aluminum Bifunctional Spatial Light Modulator", Proc. of the 48th IEEE International Midwest Symposium on Circuits \& Systems (MWSCAS'05), Cincinnati, Ohio, USA, Aug 7-10, 2005, pp. 159-162.

[9] Y. Yee, J. U Bu, M. Ha, J. Choi, H. Oh, S. Lee, and H. $\mathrm{Nm}$, "Fabrication and characterization of a PZT actuated micro-mirror with two-axis rotational motion for free space optics", Proc. of IEEE MEMS Workshop 2001, pp. 317320.

[10] H. Nam, Y. Kim, S. Cho, Y. Yee and J. Bu, "Low voltage PZT actuated tilting micro-mirror with hinge structure", Conference Digest of 2002 IEEE/LEOS International Conference on Optical MEMs, 2002, pp. 8990 .
[11] S. J. Kim, Y. H. Cho, H. J. Nam, J. U. BU, "Piezoelectrically pushed rotational micro-mirrors for wideangle optical switch applications", IEEE The Sixteenth Annual International Conference on Micro Electro Mechanical Systems (MEMS'03), Kyoto, Japan, Jan. 19-23, 2003, pp. 263-266.

[12] C. Liu, "Foundation of MEMS", Chapter 7: "Piezoelectric Sensing and Actuation", Prentice Hall, Sept. 5, 2005, ISBN: 0131472860. 